\title{
Direct Simulations of Primary Atomization in Moderate-Speed Diesel Fuel Injection
}

\author{
K. Mehravaran
}

\begin{abstract}
Our recent "Direct" simulations of liquid fuel atomization in high-pressure diesel engine injectors are presented. An adaptive volume of fluid (VOF) method based on octree meshing is used, providing savings of two orders of magnitude in computational cost. Both unperturbed and perturbed inlet conditions are used, and the results are compared to the experimental measurements of Hiroyasu \& Kadota. The overall agreement between the volumetric distribution of droplet diameter in our first trial (unperturbed) and the measurements from the injector far-field is very promising. The results of the perturbed simulation however, show a substantially smaller size distribution. This is attributed to the residual effect of the perturbation on the droplet, perhaps expediting the secondary atomization process. Detailed analysis of resolution requirements, interfacial instabilities, and tracking of droplets are underway to provide a complete explanation of the physical phenomena occuring. The simulations will be extended to other ambient densities, and later to higher-speed injectors, where different, and more complex breakup mechanisms are believed to be interacting with each other. Once these mechanisms are fully understood, it will be possible to create accurate models for implementation in Large Eddy Simulations (LES).
\end{abstract}

Index Terms-Atomization, breakup, DNS, VOF.

\section{INTRODUCTION}

A detailed knowledge of the atomization process in fuel injectors is essential for accurate modelling of the subsequent evaporation, mixing, and combustion processes. Although a detailed knowledge of surface instabilities in these injectors is still lacking, it is understood that turbu- lence in the injector and mixing layer instability in the gas phase have a significant role. The other known influence is cavitation and the collapse of vapor bubbles, affecting the turbulence in the nozzle. Simplified two-dimensional theories [1] exist, but only explaining the initial growth of the instabilities. Experimental measurements are known to suffer from a lack of resolution in the initial dense region of the spray. Moreover, in experiments, it is difficult to isolate the effects of nozzle turbulence from the interfacial instability, for a proper validation of the theories. The experimental measurements available are normally from far downstream locations, in the dilute region of the spray. The conventional approach in the numerical simulations of sprays created by fuel injectors was the application of simplified "phenomenonological" atomization models in the context of

Manuscript received January 6, 2013; revised February 28, 2013. This work was supported by The Natural Sciences and Engineering Research Council of Canada.

Author is with the University of British Columbia's School of Engineering (Okanagan). Kelowna, BC, V1V 1V7 (e-mail: kian.mehravaran@ubc.ca)
RANS [2], [3], a trend that continues to this date, speciallly in the industry. It has been followed recently in LES as well, where we [4] presented a subgrid scale model to trigger the near-field instability required for a correct jet growth rate.

Despite the advances in computer technology, the "direct" simulations of the primary breakup process have only appeared recently in a few, preliminary investigations [5]-[7]. The numerical techniques used to track the interface and calculate the surface tension accurately (VOF, level-set, or combination [5] are relatively sophisticated, and computationally demanding.

In this work, preliminary simulations of the diesel fuel primary breakup are performed and the results are discussed and compared with the experimental measurements of Hiroyasu and Kadota [8].

\section{Methodology}

The incompressible, variable-density, Navier-Stokes equations with surface tension can be written

$$
\begin{gathered}
\rho\left(\partial_{t} \mathrm{u}+\mathrm{u} \cdot \nabla \mathrm{u}\right)=\nabla p+\nabla \cdot(2 \mu \mathrm{D})+\sigma \kappa \delta_{s} \mathrm{n}, \\
\partial_{\mathrm{t}} \rho+\nabla \cdot(\rho \mathrm{u})=0, \\
\nabla \cdot u=0,
\end{gathered}
$$

with $u=(u, v, w)$ the fluid velocity, $\rho=\rho(x, t)$ the fluid density, $\mu=\mu(x, t)$ the dynamic viscosity and $D$ the deformation tensor defined as $\left.D_{i j}=\left(\partial_{i} u_{j}+\partial_{j} u_{i}\right) / 2\right)$. The Dirac distribution function $\delta_{\mathrm{s}}$ expresses the fact that the surface tension term is concentrated on the interface; $\sigma$ is the surface tension coefficient, $\kappa$ and $\mathrm{n}$ the curvature and normal to the interface.

For two-phase flows we introduce the volume fraction $(x$, $t$ ) of the first fluid and define the density and viscosity as

$$
\begin{aligned}
& \rho(c)=c \rho_{1}+(1-c) \rho_{2}, \\
& \mu(c)=c \mu_{1}+(1-c) \mu_{2},
\end{aligned}
$$

with $\rho_{1}, \rho_{2}$ and $\mu_{l}, \mu_{2}$ the densities and viscosities of the first and second fluids, respectively. The advection equation for density can then be replaced with an equivalent advection equation for the volume fraction

$$
\partial_{t} c+\nabla \cdot(c \mathrm{u})=0 .
$$

A complete description of the discretization and numerical schemes used can be found in Popinet [9]. The code used "Gerris" has been checked for conservation errors and validated [10] against the linear instability theory. One of the important aspects of the code is the octree meshing, combined with adaptive refinement. The refinements can be 
done using different criteria. Here, radius of curvature and vorticity magnitude are the refinement criteria, as an accurate prediction of both are essential to the breakup process, while the latter is also important to resolve the turbulence generated in the gas phase. The octree mesh permits a two order of magnitude savings in computational cost, making these simulations feasible. The adaptive refinement based on the octree mesh is quite cheap, and practical to perform in every time step. A cross section of the mesh in the perturbed simulation is shown in Fig. 1, where the roll-ups and regions of high-voricity can be seen (compare to Fig. 4).

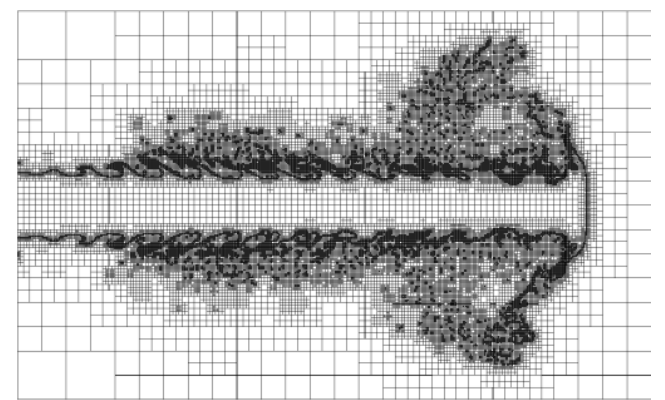

Fig. 1. A Portion of the $z=0$ cross section of the octree mesh in the perturbed simulation

\section{RESULTS}

The simulations are based on the experiments of Hiroyaso and Kadota [8], where diesel fuel is injected into a quiescent atmosphere. For this work, the gas conditions are $\mathrm{P}=$ $1.1 \mathrm{MPa}$, and $\rho=12.6 \mathrm{~kg} / \mathrm{m}^{3}$. The mean liquid jet velocity is estimated to be $102 \mathrm{~m} / \mathrm{s}$, and the injector diameter is $300 \mu \mathrm{m}$. The ratio of liquid to gas density is 66.7 , and the Reynolds and Weber numbers based on the bulk liquid conditions are 12,240 and 88,880 , respectively.

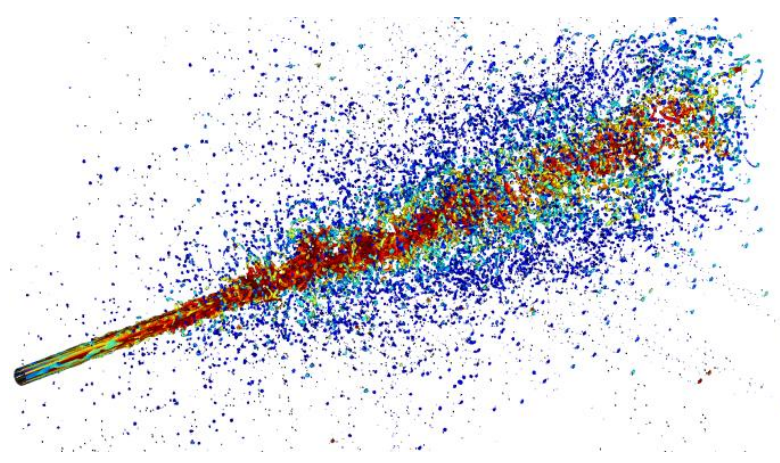

Fig. 2. Profile of the interface in the unperturbed simulation. Colours show the magnitude of the velocity on the interface.

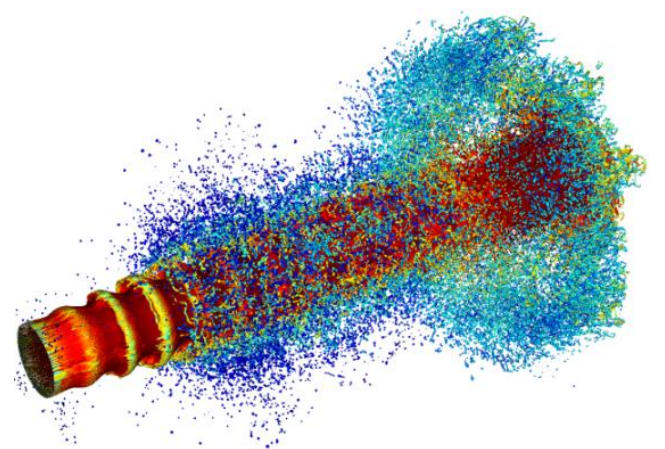

Fig. 3. Profile of the interface in the perturbed simulation. Colours show the magnitude of the velocity on the interface.
The smallest cell size used was $2 \mu \mathrm{m}$, and the simulations were performed on 4 CPUs using MPI and dynamic load balancing, with the maximum number of 10 million cells. Two simulations at the above conditions were performed; unperturbed, and perturbed (5\% amp., axial) inlet conditions. Figures 2 and 3, show the interface between the liquid and gas phases, coloured with the velocity magnitude, for the unperturbed and perturbed simulations, respectively. Interfacial instabilities are quite pronounced in the perturbed case, while the unperturbed case is without any instabilities for about 20 diameters, until it suddenly breaks up. There is also a cloud of droplets observed in the downstream region of the liquid jet tip.

The contours of vorticity magnitude for the perturbed case are shown in Fig. 4.

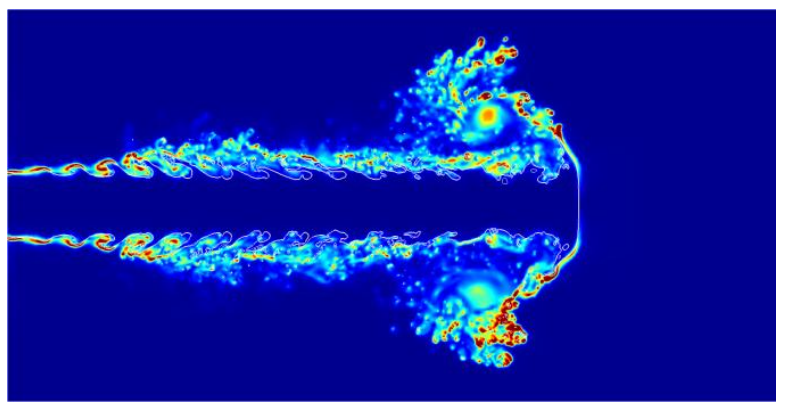

Fig. 4. Vorticity magnitude contours in the perturbed simulations. The white line shows the boundary between the phases. Cross section $z=0$ is shown.

It is seen that the liquid jet folds into a spherical tip as it impacts the quiescent, dense gas. As the jet and its tip move downstream into the gas, a highly recirculating region is left behind, triggering the breakup of the tip into droplets. This high-vorticity region is quite effective in stripping droplets away from the tip, as the tip folds back into a thin film and a cusp. The droplets broken off from the tip produce "spots" of high-vorticity as they move in the gas.

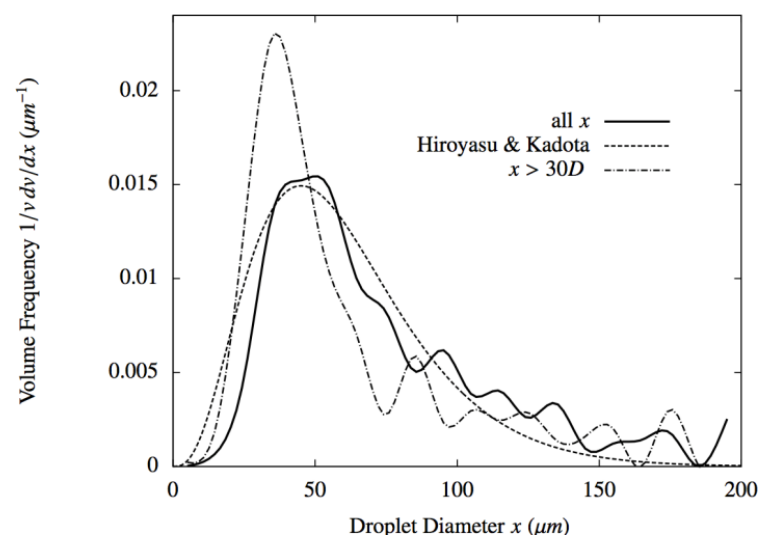

Fig. 5. Comparison of volumentric droplet size distribution with the measurements of Hiroyasu \& Kadota [3]

Droplet size distribution is compared with the measurements of Hiroyasu and Kadota in fig. 5. The distribution shown is $d v$, the incremental volume of droplets within the diameter range $x$ and $x+d x$, which can be interpreted as a volume weighted, droplet diameter distribution. To represent the experimental measurements, the Chi square distribution given [8] is used. The normalized 
form of this distribution uses the Sauter mean diameter $x_{32}$,

$$
\frac{d v}{v}=13.5\left(\frac{x}{x_{32}}\right) \exp \left(-3 \frac{x}{x_{32}}\right) d\left(\frac{x}{x_{32}}\right) \text {. }
$$

where the peak occurs at $x_{32}$.

Sauter mean diameter is defined as

$$
\sum n_{i} x_{i}^{3} / \sum n_{i} x_{i}^{2}
$$

where $n_{i}$ are the number of droplets with diameter $x_{i}$. Sauter mean diameter is the diameter of a droplet that has the same surface-to-volume ratio as that of the total liquid.

Due to the sampling technique used in the experiments, the samples are not from the vicinity of the liquid jet, and the sampled droplets have gone through a secondary break-up as well. Nevertheless, the agreement seen between the experimental sample at $75 \mathrm{~mm}$ from the injector and the overall distribution in the simulations is promising. The perturbed simulation shows a tendency towards smaller diameters (Fig. 6). While further investigations are required to find the reason to this apparent discrepancy, it is believed that the strong vortical structures created as a consequence of the perturbations, are still not fully resolved and the intermediate ligament stretching and breakup processes are mostly missed.

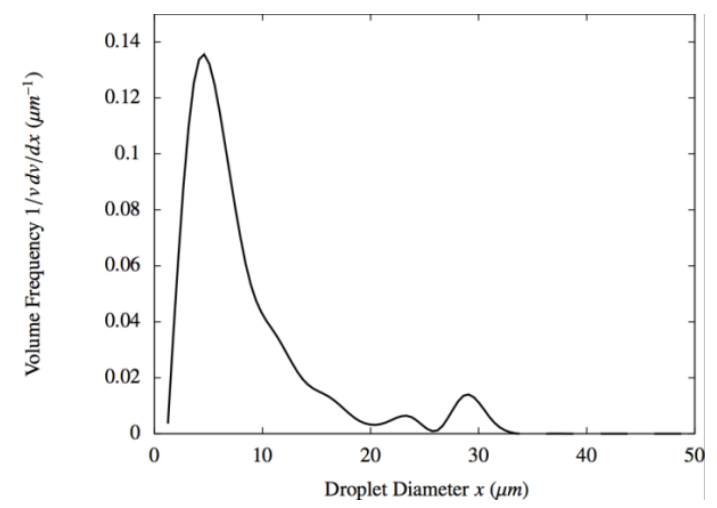

Fig. 6. Volumetric droplet size distribution for the perturbed case.

\section{CONCLUSION}

Two high-resolution simulations of diesel fuel injection into a quiescent atmosphere are performed, and compared to experimental measurements. The fuel is injected from a $300 \mu \mathrm{m}$ nozzle, at a speed of $102 \mathrm{~m} / \mathrm{s}$ into Nitrogen at 12.6 $\mathrm{kg} / \mathrm{m}^{3}$. The simulations were performed with the open-source code "Gerris", capable of adaptive octree meshing, making the simulations feasible by reducing the number of cells and computational expense.

The overall volumetric distribution of droplet diameter is in good agreement with the measurements, sampled at the injector far-field, and the chi square distribution suggested, for the unperturbed case. However, the perturbed case results show a relatively sudden breakup to very small diameters, suggesting insufficient resolution, although the perturbation-induced instabilities are known to play an important role.

Simulations with more stringent adaptive criteria are underway already, and the interfacial instabilities, and the effect of back pressure will be thoroughly investigated.

\section{REFERENCES}

[1] N. Dombrowski and W. R. Johns, "The aerodynamic instability and disintegration of vis- cous liquid sheets," Chemical Engineering Science, vol. 18, no. 3, pp. 203, 1963.

[2] T. Kaminaga, J. Kusaka, and Y. Ishii, "A three-dimensional numerical study on exhaust gas emissions from a medium-duty diesel engine using a phenomenological soot particle formation model combined with detailed chemistry," International Journal of Engine Research, vol. 9, no. 4, pp. 283-296, August 2008.

[3] T. Kaneko, T. Fujii, Y. Matsuda, and T. Chikahisa, "NOx reduction in diesel combustion by enhanced mixing of spray tip region," JSME International Journal Series B-fluids and Thermal Engineering, vol. 48, no. 4, pp. 665-670, November 2005.

[4] K. Mehravaran, Large eddy simulation in high-speed diesel fuel injection, To be submitted.

[5] T. Menard, S. Tanguy, and A. Berlemont, "Coupling level set/VOF/ghost fluid methods: Validation and application to 3D simulation of the primary break-up of a liquid jet," Int. J. of Multiphase Flow, vol. 33, no. 5, pp. 510-524, May 2007.

[6] J. Shinjo and A. Umemura, "Simulation of liquid jet primary breakup: Dynamics of lig- ament and droplet formation," International Journal of Multiphase Flow, vol. 36, no.7, pp. 513-532, July 2010.

[7] S. Popinet, "Gerris: a tree-based adaptive solver for the incompressible euler equations in complex geometries,' Journal of Computational Physics, vol. 190, no. 2, pp. 572-600, September 2003.

[8] D. Fuster, A. Bague, T. Boeck, L. Le Moyne, A. Leboissetier, S. Popinet, P. Ray, R. Scar- dovelli, and S. Zaleski, "Simulation of primary atomization with an octree adaptive mesh refinement and VOF method," International Journal of Multiphase Flow, vol. 35, no. 6, pp. 550-565, June 2009.

[9] H. Hiroyasu and T. Kadota, Fuel droplet size distribution in diesel combustion chamber, SAE Paper 740715, 1974.

[10] S. Popinet, "An accurate adaptive solver for surface-tension-driven interfacial flows," Journal of Computational Physics, vol. 228, no. 16, pp. 5838-5866, 2009.

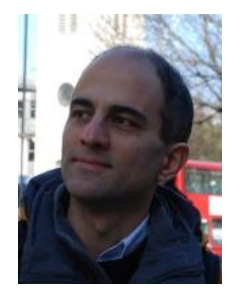

Kian Mehravaran received his Ph.D. in Mechanical Engineering from Michigan State University in 2005. His dissertation was on micro-gravity effects on turbulent flames and LES/FMDF modelling of high-speed turbulent flames with detailed chemistry. After graduation, he was as a research associate in the University of Karlsruhe, under a collaborative research program funded by the German government. He worked on understanding and modelling curvature and stretch effects on turbulent premixed flames using DNS. He has worked in air-conditioning and power-generation industries as well. Prior to joining UBC-Okanagan, he was a Research Associate in Imperial College London, where he developed models for Large Eddy Simulation (LES) of diesel fuel injection, under the project titled "LES/CMC of diesel engine combustion with detailed chemistry". 